\title{
Tuberculosis Screening Using a T-Cell Interferon- $\gamma$ Release Assay in Japanese Medical Students and Non-Japanese International Students
}

\author{
Takayuki Ogiwara, ${ }^{1}$ Takao Kimura, ${ }^{2}$ Yutaka Tokue, ${ }^{1}$ Rumi Watanabe, ${ }^{2}$ \\ Makoto Nara, ${ }^{1}$ Toshiko Obuchi, ${ }^{1}$ Akiko Yaegashi, ${ }^{3}$ Sachie Yomoda, ${ }^{1,2}$ \\ Kihachi Ohshima ${ }^{3}$ and Masami Murakami ${ }^{1,2}$ \\ ${ }^{1}$ Infection Control and Prevention Center, Gunma University Hospital, Maebashi, Gunma, Japan \\ ${ }^{2}$ Department of Clinical Laboratory Medicine, Gunma University Graduate School of Medicine, Maebashi, \\ Gunma, Japan \\ ${ }^{3}$ Gunma University Health and Medical Center, Maebashi, Gunma, Japan
}

\begin{abstract}
Screening of medical students and international students for tuberculosis (TB) at the time of admission is a key strategy to control and prevent the spread of infection on university campus and teaching hospitals because of the high risk of exposure to TB patients. The Mycobacterium tuberculosis antigen-specific T-cell interferon- $y$ release assays (IGRAs) are specific latent tuberculosis detection methods used in such groups. Currently, in Japan, there are no guidelines and no baseline data on IGRAs to evaluate the risk of TB in these high-risk groups. In order to evaluate TB risk at the time of admission in university campus and medical schools in Japan, a retrospective study was conducted. A total of 969 students (585 Japanese students and 384 international students) were screened for TB using the IGRAs at the time of admission. Eight Japanese students $(0.9 \%)$ were positive for IGRAs, but none were diagnosed with active TB at the follow-up. In contrast, 30 international students (7.8\%) were positive for IGRAs, including two students diagnosed with active TB during follow up. Positive ratio of IGRAs in international students was significantly higher than that of medical students at the time of admission. Here we propose a standard approach for TB screening with IGRAs at the time of admission for medical students and international students in Japan.
\end{abstract}

Keywords: college health; international students; medical students; T cell interferon- $\gamma$ release assay; tuberculosis screening

Tohoku J. Exp. Med., 2013 June, 230 (2), 87-91. C 2013 Tohoku University Medical Press

\section{Introduction}

To date, tuberculosis (TB) remains an important occupational risk for health-care workers because of their frequent exposure to TB patients. Therefore, screening protocols for latent TB infection (LTBI) have been implemented as a crucial infection control component in most hospitals (Pai et al. 2006; Menzies et al. 2007). In addition, screening and targeted testing are expanded to medical students as a key strategy to effectively control and prevent TB infections (Veeser et al. 2007). The reliability of the commonly used tuberculin skin test (TST) is rather limited due to the possibility of false-positives among subjects who received Bacillus Calmette-Guerin (BCG) vaccination and were exposed to non-tuberculous mycobacteria (Snider 1985). Thus, in Japan, where BCG vaccination is widely used, TST has very limited value for screening LTBI, and this necessitates the introduction of an alternative screening method. The Centers for Disease Control and Prevention (CDC) guidelines recommended replacing TST with Mycobacterium tuberculosis antigen-specific T-cell interferon- $\gamma$ release assays (IGRAs) for LTBI (Mazurek et al. 2005). Incidentally, the IGRAs were recently proposed as a standard screening protocol for medical students at the time of admission (Graham et al. 2006). According to guidelines of American College Health Association (ACHA), international students coming from countries with high TB risk should also be tested because this subpopulation has a high incidence of LTBI and high risk of developing active TB (ACHA Guidelines 2011). These IGRAs were also recommended for TB screening and targeted testing of college and university students as well (Graham et al. 2006; ACHA Guidelines 2011). In Gunma University, TSTs were replaced with IGRAs for Japanese medical stu-

Received February 21, 2013; revised and accepted May 24, 2013. Published online June 12, 2013; doi: 10.1620/tjem.230.87. Correspondence: Takao Kimura, Department of Clinical Laboratory Medicine, Gunma University Graduate School of Medicine, 3-3915 Showa-machi, Maebashi, Gunma 371-8511, Japan. e-mail: tkimura@med.gunma-u.ac.jp 
dents in 2010. In addition, we introduced IGRAs as TB screening for international students in Gunma University in 2009.

A retrospective study was conducted in 2009-2011 at the Gunma University to compare the risks of TB infection among Japanese medical students and non-Japanese international students using IGRAs, which were performed annually at the time of admission. Moreover, this study provides the first baseline data regarding IGRAs to evaluate the risk of TB in this high-risk Japanese population group. This study strongly suggests that annual screenings including international students might considerably improve the prevention and control of TB infection on university campuses worldwide.

\section{Methods}

Subjects

The present study investigated two groups of students, screened once for TB at the time of admission to the course. The first group consisted of 585 Japanese medical students of the Gunma University underwent IGRAs screening in 2010-2011. The participants were 220 medical students, 181 nursing students, and 184 paramedical students. The second group included 384 non-Japanese students underwent IGRAs screening in October 2009-2011. The international students were admitted to the department of education, engineering, social and information studies, and health science or medicine. IGRAs positive students were offered evaluation by infectious disease physicians to test for active TB by chest radiography and chest computed tomography (CT). IGRAs intermediate students were followed by chest radiography once a year. They had not been diagnosed with active TB. Three hundred and sixty-three of the 384 (94.5\%) international students were from high TB risk countries, designated on the basis of the World Health Organization (WHO) classification, namely Europe, Asia, as well as North and South America. In the both groups, age, gender, nationality and the results of IGRAs were analyzed retrospectively. This study was approved by the Ethics Committee of Gunma University Graduate School of Medicine.

IGRAs

We employed QFT-GIT as IGRAs to test TB infection. QFTGIT was performed according to the manufacturer's instructions (Cellestis Ltd, Carnegie, Australia). Results were considered positive when the TB antigen response minus the negative control responses were $\geq 0.35$ international units $\mathrm{IU} / \mathrm{ml}$, and they were considered negative when the difference was $<0.10 \mathrm{IU} / \mathrm{ml}$. Intermediate results corresponded to differences in the range of $0.10-0.35 \mathrm{IU} / \mathrm{ml}$.

\section{Statistical analysis}

Data are presented as a mean \pm standard deviation (s.D.). Categorical variables are presented as numbers and percentages. The distributions of IGRAs positive, intermediate, and negative subjects between genders or the two groups were compared of difference by using the Fisher's exact tests. Statistical significance was established for $p$-values $<0.05$.

\section{Results}

The Japanese medical students and the international students exhibited comparable age ranges, with average of $19.9 \pm 2.3$ years and $24.4 \pm 3.7$ years, respectively (Table 1 ). With regard to TB infection, IGRAs assay identified TB positive Japanese medical students each year, corresponding to $0.7 \%$ of the group in 2010 and $1.0 \%$ of the group in 2011. This screening method identified considerably more TB positive students among the non-Japanese international

Table 1. Responses of IGRAs in medical students and international students.

\begin{tabular}{|c|c|c|c|c|}
\hline & \multicolumn{2}{|c|}{ Medical students } & \multicolumn{2}{|c|}{ International students } \\
\hline Mean age, y $( \pm$ S.D. $)$ & \multicolumn{2}{|c|}{$19.9( \pm 2.3)$} & \multicolumn{2}{|c|}{$24.4( \pm 3.7)$} \\
\hline \multicolumn{5}{|l|}{ Gender } \\
\hline \multirow[t]{2}{*}{ Male/Female } & \multicolumn{2}{|c|}{187 / 398} & \multicolumn{2}{|c|}{$200 / 184$} \\
\hline & Male & Female & Male & Female \\
\hline \multirow[t]{2}{*}{ IGRAs } & $n=187$ & $n=398$ & $n=200$ & $n=184$ \\
\hline & $n(\%)$ & $n(\%)$ & $n(\%)$ & $n(\%)$ \\
\hline Positive & $1(0.5 \%)$ & $4(1.0 \%)$ & $13(6.5 \%) *$ & $14(7.6 \%) *$ \\
\hline Intermediate & $1(0.5 \%)$ & $3(0.8 \%)$ & $13(6.5 \%) *$ & $12(6.5 \%) *$ \\
\hline Negative & $185(99.0 \%)$ & $391(98.2 \%)$ & $174(87.0 \%)$ & $158(86.9 \%)$ \\
\hline \multirow[t]{2}{*}{ IGRAs } & \multicolumn{2}{|c|}{$n=585$} & \multicolumn{2}{|c|}{$n=384$} \\
\hline & \multicolumn{2}{|c|}{$n(\%)$} & \multicolumn{2}{|c|}{$n(\%)$} \\
\hline Positive & \multicolumn{2}{|c|}{$5(0.9 \%)$} & \multicolumn{2}{|c|}{$30(7.8 \%) *$} \\
\hline Intermediate & \multicolumn{2}{|c|}{$5(0.9 \%)$} & \multicolumn{2}{|c|}{$25(6.5 \%) *$} \\
\hline Negative & \multicolumn{2}{|c|}{$575(98.2 \%)$} & \multicolumn{2}{|c|}{$329(85.7 \%)$} \\
\hline
\end{tabular}

Data are presented as a mean \pm standard deviation (s.D.). Categorical variables are presented as numbers and percentages. The distributions of IGRAs positive, intermediate, and negative subjects between genders or the two groups were compared of difference by using the Fisher's exact tests. Statistical significance was established for $\mathrm{p}$ values $<0.05$. $*: p<0.01$. 
students, with a range of $4.3 \%-8.6 \%$ for the three years of screening (Table 1). Twenty-eight of the 30 (93.3\%) IGRAs positive international students were from high TB incidence countries designated by WHO classification, whereas two students were from low TB incidence countries. Positive ratio of IGRAs in Japanese medical students was significantly lower $(p<0.01)$ than that of non-Japanese international students in Gunma University, although Japan is a high TB incidence country, as designated by WHO classification. Nationalities of 384 international students were summarized in Table 2. According to WHO classification, 363 international students came from high TB incidence countries and 21 international students came from low TB incidence countries (Table 2). Among 363 students from high TB incidence countries, 28 students $(7.7 \%)$ were IGRAs positive, 24 students $(6.6 \%)$ were IGRAs intermediate and 311 students (85.7\%) were IGRAs negative. Among 21 international students from low TB incidence countries, two students $(9.5 \%)$ were IGRAs positive, one student $(4.8 \%)$ was IGRAs intermediate and 19 students $(85.7 \%)$ were IGRAs negative. IGRAs positive ratio in foreign male students was significantly higher than that of
Japanese male students (Table 1). Positive ratio of IGRAs in foreign female students was significantly higher than that of Japanese female students (Table 1). There was no statistically significant difference with regard to gender in the distribution of IGRAs responses in both the groups.

All the IGRAs positive students (5 Japanese medical students and 30 international students) underwent chest radiography and chest $\mathrm{CT}$, and they were examined for activities that might result in increased risk including volunteering, conducting research, mentoring, studying abroad, traveling, visiting relatives, or employment involving close contact with individuals in areas with increased TB incidence. None of the 5 Japanese medical students had history of TB or active TB disease at the follow-up. They were not advised LTBI therapy by infectious disease physicians. Although none of the 30 IGRAs positive international students were diagnosed with active TB at the time of admission, 2 international students $(6.7 \%)$ had active TB disease at the follow-up. Sputum examinations of cultures and PCR performed in them were negative for M. tuberculosis. They were treated by standard anti-tuberculosis drugs. They were both from high TB incidence countries, as desig-

Table 2. Nationalities of 384 international students in 2009-2011.

\begin{tabular}{|c|c|c|c|}
\hline \multicolumn{2}{|c|}{ High TB incidence countries } & \multicolumn{2}{|c|}{ Low TB incidence countries } \\
\hline Nationality & Number & Nationality & Number \\
\hline Azerbaijan & 5 & Australia & 1 \\
\hline Bangladesh & 4 & Egypt & 1 \\
\hline Brazil & 2 & Hungary & 1 \\
\hline Cambodia & 4 & Iran & 1 \\
\hline China & 156 & Italy & 3 \\
\hline Colombia & 4 & Lebanon & 1 \\
\hline Congo & 1 & Saudi Arabia & 2 \\
\hline India & 1 & Slovenia & 3 \\
\hline Indonesia & 29 & Sweden & 1 \\
\hline Kenya & 1 & United Kingdom & 1 \\
\hline Lao People's Democratic republic & 1 & United States of America & 5 \\
\hline Malaysia & 35 & Total & 21 \\
\hline Mongolia & 11 & & \\
\hline Nepal & 5 & & \\
\hline Nicaragua & 2 & & \\
\hline Nigeria & 1 & & \\
\hline Pakistan & 1 & & \\
\hline Peru & 4 & & \\
\hline Philippines & 3 & & \\
\hline Republic of Korea & 17 & & \\
\hline Russian Federation & 1 & & \\
\hline Sri Lanka & 1 & & \\
\hline Taiwan & 29 & & \\
\hline Thailand & 15 & & \\
\hline Viet Nam & 30 & & \\
\hline Yemen & 1 & & \\
\hline Total & 363 & & \\
\hline
\end{tabular}


nated by WHO classification. The other two students had undergone isoniazid therapy in their own country before they traveled to Japan, which were also high TB incidence countries. The other 2 international students were advised LTBI therapy and accepted. The other 24 foreign students were not advised LTBI treatment by infectious disease physicians. The ratio of clinical breakdown to active TB during follow-up in IGRAs positive Japanese medical students $(0 \%)$ was significantly lower $(p<0.01)$ compared with that of non-Japanese international students (6.7\%) at the Gunma University.

\section{Discussion}

In 2011, ACHA provided guidelines for TB screening and targeted testing for TB among college and university students. These guidelines stipulated that early detection provides opportunities to improve the health of the affected individuals and to reduce the potential of disease spreading as well (ACHA Guidelines 2011). Using these guidelines, high-risk populations for LTBI or TB were identified through epidemiological and population-based studies. Medical students and international students from countries with high-incidence of TB were classified as high-risk populations (ACHA Guidelines 2011). Therefore TB screening of medical students would provide a baseline for annual assessment of health-care workers exposed to TB patients; whereas screening of international students from high TB incidence countries would provide an opportunity for preventive therapy. Currently, in Japan, there are no baseline data on IGRAs to evaluate the risk of TB in these population groups. Accordingly, the present study determined the baseline immune status of Japanese medical students and non-Japanese international students at the time of admission.

In Gunma University, most international students (94.5\%) were from countries with high TB incidence rates, which may explain their significantly high positive ratios for IGRAs compared with the Japanese medical students. In this study, 363 international students came from TB high incidence countries (Table 2). Among 363 international students, 28 students $(7.7 \%)$ were IGRAs positive. The twenty-eight students were admitted to the education, engineering, social and information studies, and health science or medicine. These data are consistent with the higher positive ratios for IGRAs reported for international students as compared with medical students in Singapore (4.3\%) (Chee et al. 2009). On the other hand, the low percentage of LTBI-positive Japanese medical students $(<1 \%)$ was consistent with a survey conducted in German nursing students (Schablon et al. 2011). However, Germany is classified by WHO as a country with low TB incidence, whereas Japan is considered as a high-risk country. Recent report showed that positive ratio of IGRAs of medical students in The United Arab Emirates (UAE) was 8\% (Sheek-Hussein et al. 2012), which was comparable to those of medical students in Singapore (Chee et al. 2009), which are low-risk and high-risk countries, respectively. Hence, the WHO classification of the countries is not necessarily predictive of the incidence of TB or LTBI among medical students. Among the international students, $93.3 \%$ LBTI-positive students were from high TB incidence countries. Interestingly, the two LBTI-positive students from low-incidence populations were health-care workers in their own country. Therefore, they were high-risk subjects for TB. These data suggest that international students should be screened for TB in Japan at the time of admission, regardless of their country of origin.

Another important distinction between the two student populations is that TB positive students were only detected at the follow-up among the non-Japanese international students, with an incidence rate of $6.7 \%$. Furthermore, two other TB positive international students had undergone isoniazid therapy for active TB in their country before they arrived in Japan. These results suggest that early screening of international students coming from countries with highincidence of TB disease could potentially prevent the spread of the disease. The IGRAs responses were not affected by gender, as previously reported (Nienhaus et al. 2008; Schablon et al. 2010).

The present study distinguished three levels of IGRAs responses using QFT-GIT assays: positive $(\geq 0.35 \mathrm{IU} / \mathrm{mL})$, intermediate $(0.10-0.35 \mathrm{IU} / \mathrm{mL})$, and negative $(<0.10 \mathrm{IU} /$ $\mathrm{mL}$ ). Fong et al. (2012) reported that IGRAs testing was successfully implemented in a TB control program as a part of pre-employment screening, and most TB positive subjects accepted LTBI treatment after counseling with an infectious disease physician. They state that the manufacturer's cut-off value of $0.35 \mathrm{IU} / \mathrm{mL}$ might be too low and might lead to over diagnosis of new TB infections or TB patients undergoing isoniazid therapy. In order to facilitate the detection of these subsets of patients, Fong et al. (2012) proposed the appropriate cut-off value to be $>1 \mathrm{IU} / \mathrm{mL}$.

The present study analyzed the medical history and activities of the TB positive students in order to identify the predominant risk factors. Five IGRAs positive Japanese medical students and 26 IGRAs positive international students had no close contact with anyone diagnosed with active TB, and had not been diagnosed with active TB during their follow-up. Currently, they are being carefully monitored for any clinical symptoms of active TB.

This is the first report documenting baseline IGRAs values in medical students at the time of admission in Japan. IGRAs might constitute as an effective pre-employment TB screening tool for medical students (Veeser et al. 2007; Chee et al. 2009; Schablon et al. 2011; Fong et al. 2012; Sheek-Hussein et al. 2012). On the other hand, the updated 2010 guidelines of $\mathrm{CDC}$ and other reports stipulate that more research is needed to optimize the serial testing conditions of IGRAs for LTBI screening (Mazurek et al. 2010; Fong et al. 2012; Rangaka et al. 2012). Despite apparent limitations of IGRAs screening for LTBI, our data suggest that a standard IGRAs protocol should be imple- 
mented for screening of both medical students and international students at the time of admission to control and prevent $\mathrm{TB}$ infection on college and university campuses, including medical schools and teaching hospitals.

\section{Acknowledgements}

We are deeply indebted to Drs. Hiroyuki Sumino, Tadashi Morimura, Osamu Araki, Katsuhiko Tsunekawa and Tomoyuki Aoki for useful discussion.

\section{Conflict of Interest}

The authors declare no conflict of interest.

\section{References}

ACHA Guidelines. Tuberculosis Screening and Targeted Testing of College and University Students.

http://www.acha.org/Publications/docs/ACHA Tuberculosis Screening_Apr2011.pdf [Accessed: May 22, 2013]

Chee, C.B., Lim, L.K., Barkham, T.M., Koh, D.R., Lam, S.O., Shen, L. \& Wang, Y.T. (2009) Use of a T cell interferongamma release assay to evaluate tuberculosis risk in newly qualified physicians in Singapore healthcare institutions. Infect. Control Hosp. Epidemiol., 30, 870-875.

Fong, K.S., Tomford, J.W., Teixeira, L., Fraser, T.G., van Duin, D., Yen-Lieberman, B., Gordon, S.M. \& Miranda, C. (2012) Challenges of Interferon-gamma Release Assay Conversions in Serial Testing of Health Care Workers in a Tuberculosis Control Program. Chest, 142, 55-62.

Graham, M., Howley, T.M., Pierce, R.J. \& Johnson, P.D. (2006) Should medical students be routinely offered BCG vaccination? Med. J. Aust., 185, 324-326.

Mazurek, G.H., Jereb, J., Lobue, P., Iademarco, M.F., Metchock, B. \& Vernon, A. (2005) Division of Tuberculosis Elimination, National Center for HIV, STD, and TB Prevention, Centers for Disease Control and Prevention (CDC). Guidelines for using the QuantiFERON-TB Gold test for detecting Mycobacterium tuberculosis infection, United States. MMWR Recomm. Rep., $\mathbf{5 4}, 49-55$.
Mazurek, G.H., Jereb, J., Vernon, A., LoBue, P., Goldberg, S. \& Castro, K. (2010) IGRA Expert Committee; Centers for Disease Control and Prevention (CDC). Updated guidelines for using Interferon Gamma Release Assays to detect Mycobacterium tuberculosis infection - United States, 2010. MMWR Recomm. Rep., 59, 1-25.

Menzies, D., Joshi, R. \& Pai, M. (2007) Risk of tuberculosis infection and disease associated with work in health care settings. Int. J. Tuberc. Lung Dis., 11, 593-605.

Nienhaus, A., Schablon, A., Bâcle, C.L., Siano, B. \& Diel, R. (2008) Evaluation of the interferon-gamma release assay in healthcare workers. Int. Arch. Occup. Environ. Health, 81, 295-300.

Pai, M., Joshi, R., Dogra, S., Mendiratta, D.K., Narang, P., Kalantri, S., Reingold, A.L., Colford, J.M. Jr., Riley, L.W. \& Menzies, D. (2006) Serial testing of health care workers for tuberculosis using interferon-gamma assay. Am. J. Respir. Crit. Care Med., 174, 349-355.

Rangaka, M.X., Wilkinson, K.A., Glynn, J.R., Ling, D., Menzies, D., Mwansa-Kambafwile, J., Fielding, K., Wilkinson, R.J. \& Pai, M. (2012) Predictive value of interferon- $\gamma$ release assays for incident active tuberculosis: a systematic review and metaanalysis. Lancet Infect. Dis., 12, 45-55.

Schablon, A., Harling, M., Diel, R. \& Nienhaus, A. (2010) Risk of latent TB infection in individuals employed in the healthcare sector in Germany: a multicentre prevalence study. $B M C$ Infect. Dis., 10, 107.

Schablon, A., Diel, R., Diner, G., Anske, U., Pankow, W., Ringshausen, F.C. \& Nienhaus, A. (2011) Specificity of a whole blood IGRA in German nursing students. BMC Infect. Dis., 11, 245.

Sheek-Hussein, M., Hashmey, R., Alsuwaidi, A.R., Al Maskari, F., Amiri, L. \& Souid, A.K. (2012) Seroprevalence of measles, mumps, rubella, varicella-zoster and hepatitis A-C in Emirati medical students. BMC Public Health, 12, 1047.

Snider, D.E. Jr. (1985) Bacille Calmette-Guérin vaccinations and tuberculin skin tests. JAMA., 253, 3438-3439.

Veeser, P.I., Smith, P.K., Handy, B. \& Martin, S.R. (2007) Tuberculosis screening on a health science campus: use of QuantiFERON-TB Gold Test for students and employees. J. Am. Coll. Health, 56, 175-180. 